\title{
NONOSCILLATION PROPERTIES OF A NONLINEAR DIFFERENTIAL EQUATION
}

\author{
MICHAEL E. HAMMETT
}

Abstract. Sufficient conditions are given for the approach to zero of all nonoscillatory solutions of $\left(p(t) x^{\prime}\right)^{\prime}+q(t) g(x)=f(t)$. The conditions are related to an oscillation theorem of N. P. Bhatia concerning the equation $\left(p(t) x^{\prime}\right)^{\prime}+q(t) g(x)=0$.

Call a function on $[a,+\infty)$ oscillatory if it has arbitrarily large zeros. Otherwise call it nonoscillatory.

Consider the differential equation

$$
\left(p(t) x^{\prime}\right)^{\prime}+q(t) g(x)=0
$$

where $p(t), q(t) \in C[0,+\infty), p(t)>0, g(x) \in C(-\infty,+\infty)$.

Bhatia [1] has proved the following result.

TheOREM. All solutions of $(1)$ defined on $[0,+\infty)$ are oscillatory on $[0,+\infty)$ provided the following conditions hold:

$$
\begin{gathered}
\int_{0}^{+\infty} \frac{1}{p(t)} d t=+\infty, \\
\int_{0}^{+\infty} q(t) d t=+\infty, \\
x g(x)>0 \quad \text { if } x \neq 0, \\
g^{\prime}(x) \geqq 0 .
\end{gathered}
$$

Consider now the equation

$$
\left(p(t) x^{\prime}\right)^{\prime}+q(t) g(x)=f(t)
$$

where $f(t) \in C[0,+\infty)$. Clearly the solutions of (6) are not necessarily all oscillatory even if conditions (2)-(5) are satisfied and $f(t)$ is small in a strong sense, such as $\int_{0}^{+\infty}|f(t)| d t<+\infty$. The equation $x^{\prime \prime}+x$ $=2 e^{-t}$ satisfies (2)-(5) and $\int_{0}^{+\infty} 2 e^{-t} d t<+\infty$.

However, the solution $x(t)=e^{-t}$ is nonoscillatory. Note that $x(t)$ $=e^{-t} \rightarrow 0$ as $t \rightarrow+\infty$. This simple example illustrates the main result of this paper.

Received by the editors November 30, 1970.

AMS 1970 subject classifications. Primary 34C10, 34C15; Secondary 34D05, 34E05.

Key words and phrases. Oscillatory, nonoscillatory, nonlinear, differential equation, solution, monotonic, approach zero.

Copyright (c) 1971, American Mathematical Society 
Theorem. In (6) let conditions (2), (4), and (5) be satisfied. Also assume $f(t) \in C[0,+\infty)$,

$$
\begin{aligned}
& p(t)>k>0 \\
& q(t)>k>0
\end{aligned}
$$

and

$$
\int_{0}^{+\infty}|f(t)| d t<+\infty
$$

If $x(t)$ is a nonoscillatory solution of $(6)$ on $[0,+\infty)$ then $\lim _{t \rightarrow+\infty} x(t)$ $=0$.

Proof. Let $x(t)$ be a nonoscillatory solution. Without loss of generality assume $x(t) \neq 0$ on $[0,+\infty)$. Suppose $x(t)>0$. The case $x(t)<0$ is handled similarly.

First it is shown that $\lim \inf _{t \rightarrow+\infty} x(t)=0$. Suppose not. Then there is a number $m>0$ such that $x(t) \geqq m$ on $[0,+\infty)$. By $(4)$ and (5) it follows that $g(x(t)) \geqq g(m)>0$ and thus $x(t)$ is a nonoscillatory solution of

$$
\left(p(t) z^{\prime}\right)^{\prime}+\left[q(t)-\frac{f(t)}{g(x(t))}\right] g(z)=0 .
$$

By Theorem 1 it follows that

$$
\int_{0}^{+\infty}\left[q(t)-\frac{f(t)}{g(x(t))}\right] d t \neq+\infty .
$$

Clearly (8) implies (3) and thus

$$
\limsup _{t \rightarrow+\infty} \int_{0}^{t} \frac{f(s)}{g(x(s))} d s=+\infty
$$

Since $f(t) / g(x(t)) \leqq|f(t)| / g(m)$ it follows that

$$
\lim _{t \rightarrow+\infty} \frac{1}{g(m)} \int_{0}^{t}|f(s)| d s=+\infty
$$

contradicting (9). Hence

$$
\liminf _{t \rightarrow+\infty} x(t)=0
$$

To complete the proof it needs to be shown that

$$
\limsup _{t \rightarrow+\infty} x(t)=0 .
$$


Suppose not. Then

$$
\limsup _{t \rightarrow+\infty} x(t)>k_{1}>0
$$

Let $\epsilon$ denote any positive number. By (9), (10), and (12) choose $M \geqq 0$ such that $x^{\prime}(M)=0$ and $\int_{t_{1}}^{t_{2}}|f(t)| d t<\epsilon$ if $M \leqq t_{1} \leqq t_{2} \leqq+\infty$. Suppose $t \geqq M$ and integrate (6) on $[M, t]$ obtaining

$$
p(t) x^{\prime}(t)-p(M) x^{\prime}(M)+\int_{M}^{t} q(s) g(x(s)) d s=\int_{M}^{t} f(s) d s .
$$

Since $x^{\prime}(M)=0$ and $q(s) g(x(s))>0$, then

$$
p(t) x^{\prime}(t) \leqq \int_{M}^{t}|f(s)| d s<\epsilon .
$$

Now integrate (6) on $[t, b]$ where $b \geqq t \geqq M$ is chosen such that $x^{\prime}(b)$ $=0$. Then follows similarly

$$
-p(t) x^{\prime}(t)<\epsilon
$$

Combining (13) and (14) it follows that

$$
\left|p(t) x^{\prime}(t)\right|<\epsilon \text { for } t \geqq M
$$

and thus

$$
\lim _{t \rightarrow+\infty} p(t) x^{\prime}(t)=0
$$

Now integrating $(6)$ on $[0, t]$ gives

$$
\int_{0}^{t} q(s) g(x(s)) d s=-p(t) x^{\prime}(t)+p(0) x^{\prime}(0)+\int_{0}^{t} f(s) d s
$$

and since the terms on the right converge as $t \rightarrow+\infty$ it follows that

$$
\int_{0}^{+\infty} q(t) g(x(t)) d t<+\infty .
$$

The proof will be completed by contradicting (17).

By (10) and (12) there exists an increasing sequence of numbers $\left\{t_{n}\right\}, n \geqq 0$, with the following properties:
(a) $\lim _{n \rightarrow+\infty} t_{n}=+\infty$.
(b) For each $n, x\left(t_{n}\right)>k_{1}$.
(c) For each $n \geqq 1$, there exists a number $t_{n}^{\prime}$ such that $t_{n-1}<t_{n}^{\prime}<t_{n}$ and $x\left(t_{n}^{\prime}\right)<k_{1} / 2$. 
Let $a_{n}$ be the largest number less than $t_{n}$ such that $x\left(a_{n}\right)=k_{1} / 2$, and let $b_{n}$ be the smallest number larger than $t_{n}$ such that $x\left(b_{n}\right)=k_{1} / 2$ for $n \geqq 1$. These must exist due to (18) and the continuity of $x(t)$.

It now follows that there exists a number $k_{2}>0$ such that

$$
b_{n}-a_{n}>k_{2} \text { for } n \geqq 1 \text {. }
$$

For consider the interval $\left[a_{n}, t_{n}\right]$. By the mean value theorem there exists a number $z_{n} \in\left(a_{n}, t_{n}\right)$ such that

$$
x^{\prime}\left(z_{n}\right)=\frac{x\left(t_{n}\right)-x\left(a_{n}\right)}{t_{n}-a_{n}}>\frac{k_{1}}{2\left(b_{n}-a_{n}\right)} .
$$

Now if $\lim \inf _{n \rightarrow+\infty}\left(b_{n}-a_{n}\right)=0$, it follows that $\lim \sup _{n \rightarrow+\infty} x^{\prime}\left(z_{n}\right)$ $=+\infty$. However from (7) and (16), $\lim _{t \rightarrow+\infty} x^{\prime}(t)=0$, a contradiction.

Because of the way $a_{n}$ and $b_{n}$ were chosen, $x(t) \geqq k_{1} / 2$ on $\left[a_{n}, b_{n}\right]$, and hence by (4) and (5),

$$
g(x(t)) \geqq g\left(k_{1} / 2\right)>0 \text { on }\left[a_{n}, b_{n}\right] .
$$

From (8), (19), and (20) there follows

$$
\begin{aligned}
\int_{a_{n}}^{b_{n}} q(t) g(x(t)) d t & >\int_{a_{n}}^{b_{n}} k \cdot g\left(k_{1} / 2\right) d t \\
& =k \cdot g\left(k_{1} / 2\right) \cdot\left(b_{n}-a_{n}\right)>k \cdot g\left(k_{1} / 2\right) \cdot k_{2} .
\end{aligned}
$$

Then

$$
\int_{a_{1}}^{b_{m}} q(t) g(x(t)) d t>\sum_{n=1}^{m} \int_{a_{n}}^{b_{n}} q(t) g(x(t)) d t>k \cdot g\left(k_{1} / 2\right) \cdot k_{2} \cdot m \rightarrow+\infty
$$

as $m \rightarrow+\infty$ so that $\int_{a_{1}}^{+\infty} q(t) g(x(t)) d t=+\infty$ contradicting (17). Hence $\lim _{t \rightarrow+\infty} x(t)=0$. This completes the proof of the theorem.

It should be noted that the hypothesis of the main theorem is more restrictive than that of Bhatia's theorem. In particular conditions (7) and (8) where $p(t)$ and $q(t)$ are bounded away from zero are not necessary conditions for a nonoscillatory solution of (6) to approach zero. Consider the example $\left(t^{-1} x^{\prime}\right)^{\prime}+t^{-1} x=t^{-2}+3 t^{-4}, t \geqq 1$. This equation satisfies on $[1,+\infty)$ all conditions of the main theorem except (7) and (8) but it does satisfy conditions (2) and (3) of Bhatia's theorem. It has the nonoscillatory solution $x(t)=t^{-1}$ which approaches zero as $t \rightarrow+\infty$ from which it is easily shown that all nonoscillatory solutions approach zero. 
It would be highly desirable to relax conditions (7) and (8) as much as possible and, in fact, to drop (7) and replace (8) by (3) if possible. In any event, it was shown in the proof of the main theorem that $\lim \inf _{t \rightarrow+\infty} x(t)=0$ for any positive solution $x(t)$, and this result uses only the conditions of Bhatia's theorem along with condition (9).

It should also be noted that under the conditions of the main theorem a nonoscillatory solution does not necessarily approach zero monotonically. For example, the function $x(t)=t^{-2}(\sin t+2)$ on the interval $[1,+\infty)$ is obviously positive, nonmonotonic, and approaching zero. Clearly $x(t)$ is a solution of an obvious equation $x^{\prime \prime}+x=f(t)$ satisfying the conditions of the main theorem.

\section{REFERENCES}

1. Nam P. Bhatia, Some oscillation theorems for second order differential equations, J. Math. Anal. Appl. 15 (1966), 442-446. MR 34 \#3017.

Furman University, Greenville, South Carolina 29613 